\title{
Mujeres americanas en Galdós: una construcción identitaria a partir de la preocupación pedagógica en El abuelo (1897) y El caballero encantado (1909)
}

\author{
LAURA García SÁNCHEZ \\ Universidad Autónoma de Madrid
}

Resumen: La presencia de América en la obra literaria y periodística de Benito Pérez Galdós nace de determinadas circunstancias biográficas e históricas que, a partir de 1897, motivarán la configuración de algunos de sus personajes femeninos. Entre ellos y, a nuestro juicio, destacarán Lucrecia Richmond (viuda aristócrata de origen americano-irlandés en El abuelo, 1897), Cintia-Pascuala y la Madre-Patria (presentes en El caballero encantado, 1909) en tanto que la preocupación pedagógica que las impulsa se conforma como la pieza esencial para comprender el pensamiento galdosiano sobre América y sus relaciones con España.

Palabras clave: El caballero encantado, El abuelo, Benito Pérez Galdós, pensamiento americano, regeneracionismo, preocupación pedagógica.

American women in Galdós: an identity construction from pedagogical concern in El abuelo (1897) and El caballero encantado (1909)

Abstract: The presence of America in the Galdós's literary and journalistic work starts from certain biographical and historical circumstances that, from 1897, will motivate the configuration of some of his female characters. Among them and, in our opinion, highlight Lucrecia Richmond (aristocrat widow with American and Irish origin from El abuelo, 1897), Cintia-Pascuala and Madre-Patria (presents in El caballero encantado, 1909) while the pedagogical concern that drives them is shaped as the essential piece to understand the galdosian thought about America and its relations with Spain.

Key words: El caballero encantado, El abuelo, Benito Pérez Galdós, american thought, regeneracionism, pedagogical concern. 


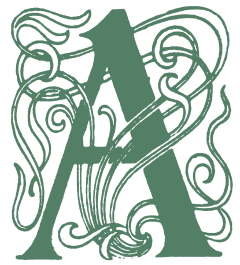

propósito de la mujer en la España de Benito Pérez Galdós, una de sus lectoras más singulares, María Zambrano, apreció que los personajes femeninos en la obra del novelista canario eran criaturas «enamoradas de su herencia, de su pasado perdido». «Ellas» - continúa la pensadora malagueña - «no pueden ser como los demás, vivir como los demás, "como los otros"». Para estas criaturas todo el mundo es " "los otros"; los otros unidos en su vulgaridad frente a la singular grandeza que las distingue. Todas luchan por su distinción, por su nobleza, princesas de inexistente dinastía, víctimas, en fin, de la historia novelera» (Zambrano, 1989: 198). Este proceso de construcción de la propia identidad como mujer y como ser humano dentro de la sociedad decimonónica española puede ser extrapolado - como establece Zambrano en su ensayo «Misericordia», publicado en la revista Hora de España en el año 1938 - a la búsqueda de la esencia de un país desvencijado $\mathrm{y}$ amenazado por las pulsiones fratricidas que se vienen sucediendo desde el comienzo de la primera guerra carlista.

Ante este desolador panorama y desde su atalaya, ¿qué propuesta de regeneración política y social podría lanzar un intelectual del reconocimiento público de Pérez Galdós? Ya en 1895, con la crisis finisecular por la pérdida de los últimos territorios coloniales acechando el destino próximo de España, el célebre protagonista de Nazarín, al ser preguntado sobre el estado actual de la conciencia y de las creencias religiosas en Europa y América, abogaba por el regreso a una Edad de Oro basada en la renuncia de toda riqueza y en la práctica de la caridad a fin de obtener una propagación efectiva y verdadera de la fe en las sociedades de ambos continentes (Pérez Galdós, 2016: 150-151). No obstante, estas ideas de don Nazario son deudoras de las de Juan Lantigua, personaje de Gloria, quien desde la opresiva Ficóbriga y en la década de 1870 advertía como única solución posible para contrarrestar los vaivenes revolucionarios que acosaban a España y Europa la unificación de las civilizaciones, amparadas siempre por un florecimiento de la fe católica ${ }^{1}$.

1 La fortuna de los Lantigua, familia paterna de Gloria, nos remite a una de las ideas más recurrentes en las referencias a América en la obra de Galdós: la del enriquecimiento y el ascenso social de los emigrados a raíz de la explotación de los recursos del continente americano. En esta primera etapa de la narrativa galdosiana será el país azteca la principal fuente de prosperidad económica: «Don Juan Crisóstomo de Lantigua nació de padres honrados en la misma villa donde acabamos de conocerle, ya gastado por la edad y consumido por el trabajo. La riqueza que desde 1860 poseía, así como la moderna casa y el bienestar tranquilo que disfrutaba, provenían de un tío suyo que volvió de Mazatlán (Méjico) con regular carga 
Fuera del territorio de la ficción narrativa, Galdós coincide con los razonamientos de sus personajes y los desarrolla en parte de su producción periodística, publicada tanto en la prensa española como en el diario bonaerense La Prensa. Tal es el caso del ensayo «Soñemos, alma, soñemos», recogido en el primer número de la revista Alma Española, que vio la luz el 8 de noviembre de $1903^{2}$. En él, nuestro escritor reflexiona acerca del pesimismo que la España anclada en el pasado pretende extender tras la catástrofe del 98 con el objeto de sugerir «la idea de un inmenso bajón de la raza y de su energía» (Pérez Galdós, 2012: 29). Frente a la corteza del mundo oficial, la propuesta de Galdós es muy clara al respecto: «necesitamos instrucción para nuestros entendimientos y agua para nuestros campos», de modo que «como el agua a los campos, es necesaria la educación a nuestros secos y endurecidos entendimientos» (Pérez Galdós, 2012: 32-33). Este proyecto pedagógico del autor de Fortunata y Jacinta parte de determinadas circunstancias culturales y biográficas como su vinculación con el pensamiento krausista y la Institución Libre de Enseñanza o la relación de naturaleza sentimental que el por entonces maduro don Benito mantuvo con la maestra Teodosia Gandarias, cuya figura parece proyectarse en la de Cintia-Pascuala, amante del protagonista de El caballero encantado.

Pero en esta tesitura cabría preguntarse de qué manera plasma Pérez Galdós dichas ideas en su producción narrativa posterior. Es aquí donde entra el universo femenino y, más concretamente, la mujer venida de ese territorio de savia nueva donde todo es posible y todo es futuro: América. El interés de Benito Pérez Galdós por el continente americano, cuyo proceso emancipatorio coincidió con la vida del escritor, se comprende a través de su labor

de pesos duros, la cual al poco tiempo soltó de sus hombros, juntamente con la de la vida, muriendo casi en el primer día de descanso. Su fortuna, que era de las más bonitas, pasó a los cuatro sobrinos, don Ángel, a la sazón capellán de Reyes Nuevos, don Juan, abogado de mucha fama, y los más jóvenes don Buenaventura y Serafinita Lantigua» (Pérez Galdós, 2006a: 221-222). Sin embargo, el pensamiento de Lantigua padre parece constituir un vaticinio de las desgracias finiseculares al tiempo que, en palabras del narrador, patrocina el retorno a una religiosidad que sirviese de consuelo a los más desfavorecidos para lograr con ello «la dispersión la esclavitud, hasta que una nueva florescencia de la fe católica en los corazones fecundados por la desgracia reorganizase a los pueblos, congregándolos bajo el manto tutelar de la Iglesia» (Pérez Galdós, 2006a: 223).

2 José Luis Mora resume las ideas más importantes del programa didáctico galdosiano mediante un decálogo en el que conviven los siguientes planteamientos: el respeto a la tradición y a la raza; el rechazo a la pobreza, a la burocracia o al socialismo obrero; la superación del pesimismo que atacaba España; la renovación de las actividades agrícolas e industriales; o, finalmente, la confianza en la cultura española, así como en la reforma moral, política y económica que promovía el institucionismo (1993: 741-742). 
literaria y periodística y, sobre todo, gracias a sus circunstancias familiares. A partir de 1898 se incrementa esta presencia de la cuestión americana en la obra del novelista, tal y como se refleja en los Episodios Nacionales Prim y La vuelta al mundo en la Numancia o en El caballero encantado. Empero, la novela en cinco jornadas El abuelo, publicada en 1897, parece marcar la intersección entre la etapa colonial y la etapa poscolonial en las que podría dividirse la amplia producción galdosiana. En este drama nos encontramos con Lucrecia, personaje de procedencia americana-irlandesa que será víctima de la incomprensión y del rechazo que su estilo de vida provoca en la nobleza decadente representada por el conde de Albrit. Por otra parte, en El caballero encantado, la construcción de la identidad iberoamericana y el futuro de sus relaciones con España se bifurca en el impulso pedagógico y regeneracionista encarnado por Cintia-Pascuala y por la simbólica Madre-Patria. La mujer se convierte así en elemento configurador en aras de interpretar el pensamiento sobre América de Galdós.

El retrato de esa identidad femenina americana comienza a esbozarse, como acabamos de referir, con Lucrecia Richmond, controvertida condesa de Laín a la que se asigna el papel antagonista al de don Rodrigo, conde de Albrit y señor de Jerusa y de Polán. Hija de un irlandés establecido en los Estados Unidos y trasladado a España por los negocios petrolíferos, la americana-irlandesa contrajo matrimonio con Rafael, único hijo de Rodrigo de Arista, cuyo glorioso linaje lo emparenta con un antiguo virrey de Perú y le hace justo beneficiario de las minas de Hualgayoc. Como consecuencia de su condición foránea y de sus licenciosas costumbres, pues actúa con gran libertad de movimientos pese a su maternidad y a su posición de viuda de la aristocracia española, Lucrecia es sometida a todo tipo de juicios negativos por parte de los demás personajes y acusada de no haber adoptado las convenciones vitales españolas. Así, el primer conocimiento que el lector/ espectador galdosiano tenga de la dama será a través de la boca de Gregoria (mujer de Venancio, actual propietario de La Pardina), para quien la madre de Nell y Dolly no es más que una «ruin pécora» y una «condenada extranjera, de quien se enamoró como un tontaina» Rafael, el malogrado hijo de don Rodrigo (Pérez Galdós, 1986: 15). Sin embargo, el conde de Albrit, a pesar de su animadversión manifiesta hacia la viuda de su heredero, deja entrever el cariño que el pueblo de Jerusa profesa a la americana: «no sé quién es peor: si tú, que paseas impune por el mundo tu desvergüenza, o un pueblo servil 
y degradado que te festeja y adula» (Pérez Galdós, 1986: 54). Esta primera aproximación a la nuera del Conde se ve completada por Senén, antiguo criado de la casa Laín y actual empleado de la Condesa, personaje que no solo defiende las diversiones de la viuda ante la moralidad imperante, sino que también la reviste de virtudes como «un corazón tierno y una voluntad generosa y franca hasta dejárselo de sobra», esto es, mujer que "compadece a los desgraciados y consuela a los afligidos», definiéndola además como «persona de instrucción» que llega a dominar hasta cuatro lenguas (Pérez Galdós, 1986: 23).

Por su parte, la simpatía de Galdós hacia el personaje de Lucrecia se revela en la extensa acotación descriptiva con la que se da inicio a la segunda jornada de la obra. En ella, nuestro narrador teatral nos la dibuja como «mujer hermosa, de treinta y cuatro años, del tipo que comúnmente llamamos interesante, mezcla feliz de belleza, dulzura y melancolía: Castaño el cabello, el rostro alabastrino, de un perfil elegante, precioso modelo de raza anglo-sajona, recriada en América» (Pérez Galdós, 1986: 55). Tal vez la mencionada orientación favorable de nuestro autor venga dada por algunos de los antecedentes familiares que pudieron contribuir en la configuración social y psicológica de Richmond. Como señala Pedro Ortiz-Armengol, la procedencia americana-irlandesa de Lucrecia nos retrotrae a la americana-escocesa Adriana Tate, pareja sentimental de José María Galdós, tío materno del escritor que se trasladó a Trinidad (Cuba) por su puesto de síndico-procurador ${ }^{3}$. La figura de Tate, denostada por Dolores Galdós (hermana de José María y madre de Benito) durante toda su vida, cobra especial relevancia al conocer que de los amores entre el tío del escritor y la americana afincada en Cuba nació una hija ilegítima que jamás sería aceptada por el resto de la familia. Esta hija, llamada María Josefa Wassinton de Galdós y apodada en Las Palmas como Sisita, fue el primer amor del adolescente Benito y causa fundamental de una resolución materna que marcaría el devenir vital del joven: su traslado forzoso a Madrid para cursar los estudios universitarios de Derecho y olvidar así los inapropiados amores con la prima ilegítima4 (Ortiz-Armengol, 2000:

3 La figura de Domingo de Galdós y Alcorta, abuelo materno del escritor, constituye la rama primigenia de la que saldrán otros familiares de la importancia de José María Galdós o Benito de Galdós y Medina (De Armas, 2013: 787-788). No obstante, y como consecuencia de la estrecha relación del tío José María con Dolores, madre de Benito, nos interesan especialmente los pormenores vitales de este familiar y de sus descendientes, cuyos conflictos tendrían una trascendencia especial en la obra posterior de su sobrino.

4 Ortiz-Armengol también se hace eco de las relaciones que Berkowitz establece entre los caracteres de El abuelo y el entorno familiar galdosiano: el rechazo hacia una hija natural como Sisita o la preocupación 
28-35). El conflicto familiar que se plantea en el drama - y que atiende, en resumidas cuentas, al rechazo de una hija natural como Sisita - encuentra su momento álgido en la defensa que la viuda lleva a cabo de la legitimidad de Nell y Dolly (aunque una de ellas no sea hija biológica de Rafael):

EL CONDE. - ¿Será usted capaz de rechazar mi proposición, de desairarme, de negar lo que pide el infortunado Albrit?

LUCRECIA. - Con grandísima pena me veo precisada a negarlo. Mis hijas son mis hijas. A ellas les conviene el calor maternal, y a mí el cariño y la presencia continua de entrambas para vivir en paz con Dios, y asegurarme la rectitud de mi alma. La una es mi deber, la otra mi error. Mi conciencia necesita los dos testigos, las dos presencias, para que yo pueda tener siempre entre mis brazos, sobre mi corazón, mis buenas y mis malas acciones. (Pérez Galdós, 1986: 223)

A la fuerza del componente biográfico en las connotaciones positivas presentes en la Condesa han de unirse, sobre todo, sus contribuciones a la regeneración y al avance técnico y cultural de Laín. Su papel de bienhechora de la comarca, apenas vislumbrado con anterioridad, adquiere una dimensión pedagógica fundamental para el propósito de Galdós cuando Lucrecia acepta con entusiasmo la petición del alcalde:

LUCRECIA. - ¿Pero qué he hecho yo, señor D. José de mi alma, para estos obsequios, este entusiasmo?

LA ALCALDESA. - Hija, la carretera de Forbes, la estación telegráfica... la condonación...

LUCRECIA. - Me bastó pedírselo al Ministro...

EL ALCALDE. - Más que todo eso vale el Instituto de segunda enseñanza, que nos disputaban los de Durante. Nada agradecen tanto los pueblos, señora mía, como el que les den algo que se le quita al vecino. Cuestión de amor propio: la entidad pueblo es lo mismo que la entidad persona. Fastidiar al vecino, y caiga el que caiga. Jerusa verá siempre en la ilustre

del conde por el linaje - al igual que le sucedía a Dolores Galdós - . Asimismo, el propio Ortiz-Armengol añade otra importante concomitancia como la presencia de un abuelo, identificable con «un hombre de 56 años que era padre de una niña [...]», de modo que «quizá el asunto más vivo en el drama escrito en 1897 sea el de los afectos del viejo conde hacia las niñas, trasunto de situaciones de Benito Pérez Galdós» (2000: 356-357). 
Condesa de Laín una individualidad digna de todos nuestros respetos. Y yo, que llevo el corazón en la mano, que digo siempre la verdad llana y monda... soy así, muy bruto, muy francote... le aseguro a usted que la queremos aquí... como sabe querer Jerusa; y si lográramos que nos concedieran la Escuela de Comercio que pretenden los de Durante, no le quiero decir a usted... La apoteosis que le haríamos retumbaría en la China.

LUCRECIA. - (Sonriente.) Yo sí que no vuelvo de mi apoteosis. (Pérez Galdós, 1986: 58)

Así las cosas, la provechosa canalización de las influencias ministeriales de la dama, su empatía con las necesidades del pueblo y sus intentos por lograr una verdadera nivelación social a través de la educación y de la eliminación de los prejuicios derivados de la primacía del linaje sanguíneo avanzan la importancia que Galdós dará a la mujer de América como motor de la remodelación nacional en El caballero encantado.

Redactada en Santander durante el verano de 1909, El caballero encantado tiene como telón de fondo la convulsa situación social y política que España atravesaba debido a acontecimientos como la guerra de Marruecos, la Semana Trágica de Barcelona, el fusilamiento de Ferrer i Guàrdia o la caída de Antonio Maura. Por ello, no es de extrañar que Galdós vuelque en este cuento real inverosímil muchas de sus ideas regeneracionistas en torno a problemas como el caciquismo, el anticlericalismo y, especialmente, la cuestión agrícola y la educación ${ }^{5}$. En su tesis doctoral «Ideología y novela en Galdós», Carolina Fernández Cordero afirma que:

El caballero encantado «recoge todos los tópicos costistas tratados por Galdós previamente: el conflicto de la tierra y su actitud frente al campesino [...], la unión del pueblo y la aristocracia como una nueva manera de salvar la sociedad española [...], la libertad de cultos y el amor libre más allá de las convenciones sociales y económicas. (2014: 177)

\footnotetext{
5 A propósito de la recepción de la novela en el contexto hispanoamericano y, especialmente, de su repercusión en Cuba, se recomienda acudir a Chamberlin, a Viñalet y sus respectivos artículos «A Cuban's reply to Galdós: "El caballero encantado y la moza esquiva" » y «De cómo Fernando Ortiz supo hallar una moza esquiva para cierto caballero encantado». Esta versión «libre y americana» de Ortiz - como reza el subtítulo de la obra -, publicada por primera vez dentro de la Revista Bimembre Cubana en 1910, es claro ejemplo del impacto de las ideas galdosianas en la isla, aunque introduce algunas variaciones como «the words "y la moza esquiva" in the title and the outrageously humorous appended letters - allegedly írom two vivacious young women readers of Galdós, two sisters who have left home and are now enjoying their freedom» (Chamberlin, 1986: 67) que proporcionan una inteligente dosis de humor a la novela e implican, además, que se erija como una reivindicación de la otredad cubana. En última instancia, para Viñalet, «es grito del derecho a ser ante cualquier intento de absorción» (2000: 52).
} 
Se trataría, por tanto, de un regeneracionismo radical nacido al amparo de los sucesos mencionados anteriormente.

El aura quijotesca que envuelve el relato subraya, en parte, el carácter fantástico e incluso utópico que subyace en la configuración de los dos personajes femeninos que nos van a ocupar: Cintia-Pascuala y la Madre. Ambas vienen a ofrecer al lector un mensaje de esperanza que pasa por la unión de América y España como correlato de la indispensable alianza entre el pueblo y las clases privilegiadas en aras del progreso nacional. La dualidad identitaria presente en la configuración de los protagonistas, Tarsis-Gil y Cintia-Pascuala, refuerza la intención del mensaje galdosiano, puesto que en ellos se concretará la fusión de las dos clases y de los dos continentes con el nacimiento de su hijo Héspero. El origen americano de Cintia, «una huérfana millonaria nacida en Bogotá y recriada en la Argentina» (Pérez Galdós, 2006: 161) que rechaza las pretensiones amorosas del caprichoso aristócrata Carlos Tarsis y lo abandona por un diplomático, es desde el primer momento mecanismo proléptico del componente mágico, del prodigio (esto es, el encantamiento de Tarsis en el pastor/campesino Gil) y de la idealización que se asocian con el otro continente. De esta manera, y como puntualiza el narrador, el nombre de Cintia, «de dulce sabor pastoril y pagano» es producto de «cierta inclinación» de las americanas «a paganizar los nombres, cual si quisieran iniciar una sombría escapada de las sombrías esferas del cristianismo» (Pérez Galdós, 2006b: 161-162). La obsesión de Tarsis por la hermosa colombiana lo conduce a una serie de fenómenos extraordinarios y sugestivos que desembocan en su propia transformación en pastor. A modo de ejemplo, el enamorado caballero cree ver a su amada al otro lado del espejo o se figura el encantamiento de su casa.

Pero la imagen ilusoria y voluble que Cintia muestra en su primera figura obtiene un peso ideológico fundamental cuando la rica heredera tome el cuerpo de Pascuala, maestra de primeras letras cuya timidez y modestia se oponen al carácter expansivo de la americana. El encuentro casual con Tarsis - ya convertido en Gil - en una fuente nos remonta al simbolismo erótico-amoroso medieval (Rodríguez Puértolas, 2006: 221), por lo que la relación que se desarrolle entre los dos protagonistas estará plagada de muchos de los códigos de la literatura pastoril y de caballerías, reminiscencias que se ilustran a partir de escenas como aquella en la que «sin decir nada, la lindísima mujer alzó el cántaro y lo inclinó sobre su brazo izquierdo para que el sediento bebiese» (Pérez Galdós, 2006b: 221). 
La instrucción de Pascuala, formada como maestra en Zaragoza, explica que la única ambición de la joven sea dirigir una escuela de párvulos en algún pueblo de la comarca de Ágreda (Soria). Es precisamente la irrupción de este alter ego femenino consagrado al magisterio el que hace de la escuela rural de la Restauración el eje estructural del relato, situándolo así en la línea de las novelas regeneracionistas de la época6 (Ezpeleta Aguilar, 2009: 205), elevadas hasta la categoría de género narrativo gracias a su recurrencia temática: el análisis de los males de la patria asociados a una institución esencial y básica como la escuela. Asimismo, los humildes orígenes familiares de Pascuala propician que su proyecto de vida condense en sí mismo el lema costista «escuela y despensa ${ }^{7}$ » (con el que Galdós ya se había identificado en el ensayo de 1903), dado que, como ella misma relata a Gil:

- Vivo con mis tíos Saturio Borjabad y su mujer Baltasara, y esta casita es de unos primos míos por parte de madre, llamados aquí los Almuerzos, porque son de la sierra de este nombre y se dedicaban al negocio del carbón. Ahora viven en Soria. Mi madre se llamaba Pilar Arabiana; dicen que era un poquito noble. Mis tíos los Borjabades tienen en Suellacabras dos o tres telares, y allí viven mis primos, que fabrican sayas y capotillos de jerga. Conque ya tienes ante ti todo el mapa de mi familia. Al ponértelo delante, me río como ves... En mi parentela hubo nobles y plebeyos; hoy todos son pobres. Algunos viven de ilusiones, otros emigran, algunos trabajan como negros... Yo, que en pobreza no tengo a nadie que me aventaje, les alegro a todos con mi alegría (Pérez Galdós, 2006b: 226).

La entrega total de Pascuala a su oficio una vez que obtenga un puesto como maestra en el municipio de Catalañazor ocasiona que su implicación emocional con los pequeños alumnos y los conflictos del pueblo aprisionen su voluntad y le impidan desligarse de su labor. Tal es la desesperación de la antigua colombiana que Gil no ve otro remedio que secuestrarla para evitar que su compañera pierda la cordura y la libertad por completo. De un modo mucho más acusado que en el caso de la condesa de Laín, el pueblo adora a Pascuala, quien ha volcado en él toda su riqueza moral y espiritual, y así se

6 Dentro de esta modalidad, cabe citar algunos títulos como La ley del embudo de Queral y Formigales (1897), Pío Cid de Ángel Ganivet (1898) o Doña Mesalina de López Pinillos (1910).

7 Costa es para Galdós su principal referente regeneracionista. De algunas de sus obras como Colectivismo agrario en España (1898), Reconstitución y europeización en España (1901) u Oligarquía y caciquismo (1902) toma don Benito ideas básicas como la importancia de la función del maestro o del árbol y el agua en la regeneración del país (Rodríguez Puértolas, 2006: 51). 
constata en el momento en el que se produce el intento de fuga con la maestra, reprimida por los niños del pueblo, que, según Rodríguez Puértolas, se encuentran en un estado de miseria y abandono que los identifica con los campesinos y los obreros de la España oficial (2006b: 56-57).

Finalmente, la devoción maternal en el trato con sus alumnos no puede sino desembocar en la propia maternidad del personaje. Héspero, hijo ya de Tarsis y Cintia y, por consiguiente, adscrito desde su nacimiento a la burguesía, estará destinado a difundir el discurso de unificación de los territorios hispanos y de las clases sociales en pos de una nueva realidad española. En definitiva, este «maestro de maestros» ha llegado al mundo gracias al vientre de Pascuala, un vientre que, como el de Fortunata, aúna la fuerza del pueblo y la corriente de la vida (Zambrano, 1989: 120). Pueblo y vida se erigen entonces como los cimientos sobre los que se edifica la tradición o, lo que es lo mismo, la raíz de nuestra cultura y de nuestra existencia.

Por último, nos gustaría referirnos brevemente al personaje de la Madre-España, guía de Tarsis en su particular encantamiento y proceso de aprendizaje y principal manifestación tanto del espacio y del tiempo mítico que se crea en el relato como de la pretensión alegórica que persigue la obra. Cabe anotar que los rasgos mitológicos de su figura - explicitados a través del uso de epítetos épicos o de su apariencia de divinidad - la equipararían, de algún modo, a otras madres galdosianas como la Mariclío de la quinta serie de los Episodios Nacionales $^{8}$ (Behiels. 1998: 84). En una de sus primeras intervenciones, la misteriosa anciana se dirigirá al caballero del siguiente modo:

\footnotetext{
8 Fuera de las similitudes iniciales, el estudio de Behiels se encuentra destinado a desmontar la identificación espontánea de ambas, extendida a raíz del Ensayo de un censo de personajes galdosianos de Sáinz de Robles y respaldada recientemente por Sadi Lakhdari en "La Madre y Mariclío, personificaciones de España en la obra de Benito Pérez Galdós”, incluido en Tomás Ferré, F. (2011), Miradas Sobre España, págs. 215-220. Desde la lectura defendida por Behiels, se establecen cuatro diferencias fundamentales entre Mariclío y la anciana madre de El caballero: la denominación dispar de una y otra por parte del narrador y del resto de personajes (pues Mariclío recibe el nombre de "Madre" de manera aislada y anecdótica en el episodio Amadeo I); la definición de su naturaleza y de sus funciones (la Madre como "alma de la raza" de la España antigua y contemporánea frente a Mariclío como observadora y musa de la Historia); en tercer lugar, el carácter distintivo de sus atributos (mientras que Clío se asocia con la cultura griega, el origen de la Madre nos remonta al pasado hispánico prerromano); por último, su papel en la estructura de las novelas, puesto que la Madre se centra en su intervención dentro de la experiencia iniciática de Tarsis (por lo que muere una vez que se completa la evolución del caballero desde el parasitismo de la aristocracia hasta un activo papel en la regeneración del destino de España) y Mariclío, si bien hace lo propio con Tito en su tarea de historiador, va poco a poco desapareciendo del relato hasta quedar prácticamente al margen de los logros de su discípulo (Behiels, 1998: 84-89).
} 
Se te ata corto a la vida, para que adquieras el cabal conocimiento de ella y sepas con qué fatigas angustiosas se crea la riqueza que derrocháis en los ocios de la Corte. Verdades hay clarísimas que vosotros, los caballeretes ricos, no aprendéis hasta que esas verdades os duelen, hasta que se vuelven contra vosotros los hierros con que afligís a los pobres esclavos, labradores de la tierra, que es como decir artífices de vuestra comodidad, de vuestros placeres y caprichos. (Pérez Galdós, 2006b: 198-199)

La dureza de sus declaraciones y el compromiso social patente en las mismas nos llevan hasta Gustavo Correa, quien ya apuntó la función de este personaje femenino como la encargada de revelar a sus hijos «el alma de la raza y de la nación española» para dirigirles con ello al «camino de la regeneración y recuperación de su antiguo vigor perdido, a través de nuevos ideales y programas de vida» (1963: 14-15). Entre esos nuevos ideales y programas de vida a los que alude Correa, la Madre-España pasa a ser el heraldo que anuncia el porvenir común socialista y republicano que aguarda a España e Hispanoamérica, territorios hermanos conectados por su lengua: «Allá como aquí, domino por mi aliento, sicut tuba; por la vibración de mi lenguaje, que será el alma de medio mundo» (Pérez Galdós, 2006: 212). A caballo entre la forma humana y la sobrenatural, lo que la emparenta con los dioses de la mitología griega - con los que comparte algunas cualidades como la inmortalidad, el antropomorfismo o la ubicuidad (Fernández Cordero, 2014: 339) -, la Madre encarna en su configuración la utopía galdosiana, basada en la reconciliación con la historia y en la desaparición de la propiedad agrícola explotada en exclusiva por los terratenientes ${ }^{9}$ (Rodríguez Puértolas, 2006: 64), así como en una esperanza de unidad hispana y progreso que cerrará la vida literaria del canario. Y es que, precisamente, ese mensaje es el legado de su última obra, Santa Juana de Castilla (Mora García, 2000: 510).

De esta suerte, la Madre-España se nos presenta como la protectora por excelencia del pueblo humilde e incansable, al tiempo que se convierte también en mártir de la opresión oligárquica tras ser expulsada de su residencia -el Castillo de Clavijo - , detenida, denigrada y asesinada por la Guardia Civil ${ }^{10}$.

\footnotetext{
9 Ante la concepción determinista de la escala social que se desprende del viaje por la Soria yerma de los protagonistas, solo resta el espiritualismo que, en palabras del propio Galdós en su artículo «El $1^{\circ}$ de mayo», vendría a ser una solución que proclama «el desprecio de las riquezas, la resignación cristiana, el consuelo de la desigualdad externa por la igualdad interna, o sea, la nivelación augusta de los destinos humanos en el santuario de la conciencia».

10 Como Benigna de Casia, protagonista de Misericordia cuya trayectoria vital se identifica con Jesucristo, la Madre también padece el calvario (la expulsión, detención y escarnio público al ser arrastrada
} 
Sin embargo, el carácter simbólico del que se ha dotado al personaje posibilitará su resurrección y, con ella, el triunfo de una nueva raza fundada en el poder del pueblo y de la educación, esto es, en la recategorización de España como Hesperia, tierra de esperanza y de la hispanidad (Fernández Cordero, 2014: 343), pues los niños representados por Hesperio, como la Madre declara, «son la generación que ha de venir; son mi salud futura; son mi fuerza de mañana» (Pérez Galdós, 2006b: 289).

La sucinta semblanza de estos tres personajes femeninos nos permite concluir el compendio de las ideas regeneracionistas galdosianas en su configuración, en tanto que todas ellas presentan un compromiso pedagógico probado, una férrea defensa de las clases más desfavorecidas y del sustrato popular de la cultura española y, principalmente, una confianza en el futuro asentada en la alianza y mutua colaboración entre dos tierras hermanas: España y América. Si en Lucrecia y en Cintia-Pascuala los aires regeneracionistas surgen de sus raíces americanas y de su formación académica, la anciana Madre evoluciona desde personajes anteriores como Benigna para así reforzar la siguiente hipótesis zambraniana: el porvenir, la reasunción de la tradición, la fuerza del pueblo y, en definitiva, la vida, terminarán por triunfar simbólicamente sobre la opresión ejercida por aquellos que detentan el poder desde la intolerancia. En este sentido, la omnipresencia de la realidad educativa - bien sea mediante los donativos para la construcción de escuelas (Lucrecia), el ejercicio de la profesión de maestra (Pascuala) o las lecciones morales de la Madre a Gil/ Tarsis $^{11}$ - evidencia la preocupación pedagógica en ambas obras y es, al mismo tiempo, el cauce de expresión de la siguiente propuesta: el conocimiento de la historia como base de un pensamiento libre y la armonización de voluntades, corazón y oficio en la búsqueda de una sociedad renovada (Mora García, 1993: 742).

por los guardias en una cuerda de presos), la muerte (sacrificio redentor) y, finalmente, la resurrección (liberación del yugo, emancipación del pueblo y convivencia pacífica de todas las capas de la sociedad y de España e Hispanoamérica con el nacimiento de Hesperio). Es más: las similitudes entre Benigna y la Madre se refuerzan por su adscripción al mundo de la gleba galdosiana, espacio en el que, como expuso Zambrano «hay algo vivo, algo que es vehículo de continuidad, de pervivencia de un pasado que corroe [...]» (1994: 134).

11 Ezpeleta Aguilar, al igual que ya había hecho Peter Bly, defiende la herencia del Bildungsroman patente en el retrato caricaturesco de Tarsis, héroe intelectual de la novela (2009: 214). Uno de los argumentos favorables para aceptar dicha adscripción sería, justamente, el papel de la Madre como guía de la transformación/evolución del caballero Tarsis. 
Sea como fuere, Galdós moldea tres tipos femeninos ligados a América en los que la caridad - entendida aquí como el desprendimiento fundado en el deleite de proveer la felicidad ajena a través de la campaña educativay la superación de las adversidades raciales y económicas determinan una construcción identitaria de la mujer como ser destinado a liderar el hermanamiento definitivo de los territorios hispanos. Tal vez sean Lucrecia, Pascuala y la Madre Patria las tres mujeres de la narrativa galdosiana que mejor condensen el utópico pensamiento universalizador sobre América que, a la postre, abrazó y defendió nuestro canario más internacional.

\section{REFERENCIAS BIBLIOGRÁFICAS}

Armas, Frederick A. de (2013), «Una rama de la familia de Galdós en Cuba: genealogía e influencia», en Actas del IX Congreso Internacional Galdosiano, Las Palmas de Gran Canaria, Cabildo de Gran Canaria, págs. 787-796.

Behiels, Lieve (1998), «Diosas y madres en la obra tardía de Benito Pérez Galdós», en Derek Flitter (coord.), Actas de XII Congreso de la Asociación Internacional de Hispanistas. Del Romanticismo a la Guerra Civil, Vol. IV, Birmingham, University of Birmingham, págs. 84-91.

Chamberlin, Vernon A. (1986), «A Cuban's reply to Galdós: El caballero encantado y la moza esquiva», en Anales galdosianos, año XXI, págs. 63-67.

Correa, Gustavo (1963), «El sentido de lo hispánico en El caballero encantado de Pérez Galdós y la Generación del 98», en Thesaurus: boletín del Instituto Caro y Cuervo, $\mathrm{n}^{\circ} 18$ (1), págs. 14-28.

EzPeleta Aguilar, Fermín (2009), Maestro y formación en la novela galdosiana, Las Palmas de Gran Canaria, Ediciones del Cabildo.

Fernández Cordero, Carolina (2014), Ideología y novela en Galdós (1901-1920), tesis doctoral dirigida por Julio Rodríguez Puértolas y Carmen Valcárcel Rivera, Madrid, Universidad Autónoma de Madrid.

Mora García, José Luis (1981), Hombre, sociedad y religión en la novelística galdosiana (1888-1905), Salamanca, Ed. de la Universidad de Salamanca. 
Mora García, José Luis (1993), «Galdós novelista: a propósito de El caballero encantado», en Actas del Cuarto Congreso Internacional de estudios galdosianos (1990), Vol. 1, Las Palmas de Gran Canaria, Cabildo de Gran Canaria, págs. 731-755.

Mora García, José Luis (2000), «Galdós y el llamado "Problema de España"», en Carmen Yolanda Arencibia (ed.), VI Congreso Internacional Galdosiano 1997, Las Palmas de Gran Canaria, Cabildo de Gran Canaria, págs. 504-514.

Ortiz-Armengol, Pedro (2000), Vida de Galdós, Barcelona, Crítica.

Pérez Galdós, Benito (1986), El abuelo, Madrid, Alianza Editorial.

Pérez Galdós, Benito (2006a), Doña Perfecta. Gloria, ed. Carmen Yolanda Arencibia, Las Palmas de Gran Canaria, Cabildo de Gran Canaria.

Pérez Galdós, Benito (2006b), El caballero encantado (cuento real...inverosímil), ed. Julio Rodríguez Puértolas, Madrid, Akal.

Pérez Galdós, Benito (2012), «Soñemos, alma, soñemos», en José Esteban (ed.), La fe nacional y otros escritos sobre España, Madrid, Rey Lear, págs. 27-35.

Pérez Galdós, Benito (2016), Nazarín, Madrid, Alianza Editorial.

Rodríguez Puértolas, Julio (2006), «Estudio preliminar» a Benito Pérez Galdós, El caballero encantado (cuento real...inverosímil), Madrid, Akal, págs. 7-130.

VIÑAlet, Ricardo (2000), «De cómo Fernando Ortiz supo hallar una moza esquiva para cierto caballero encantado», en América sin nombre: boletín de la Unidad de Investigación de la Universidad de Alicante "Recuperaciones del mundo precolombino y colonial en el siglo XX hispanoamericano", $\mathrm{n}^{\mathrm{o}} 2$, págs. 43-55.

Zambrano, María (1989), La España de Galdós, Madrid, Ediciones Endymión.

Zambrano, María (1994), «Mujeres de Galdós», en Asparkía. Investigació feminista, $\mathrm{n}^{\circ} 3$, págs. 129-135. 